\title{
The gas and star formation in bulges
}

\section{David Fisher}

Department of Astronomy, University of Maryland, email: dbfisher@astro.umd.edu

\begin{abstract}
.
\end{abstract}

